\title{
Facilitated Diffusion in the Dissolution of Carboxylic Polymers
}

\author{
Duc A. Nguyen and H. Scott Fogler \\ Dept. of Chemical Engineering, University of Michigan, Ann Arbor, MI, 48109 \\ DOI 10.1002/aic.10329 \\ Published online in Wiley InterScience (www.interscience.wiley.com).
}

\begin{abstract}
Carrier-mediated transport plays an important role in the dissolution of carboxylic polymers in aqueous solutions. Experiments with a rotating disk apparatus showed that the rate of polymer dissolution increased significantly with the addition of proton-carriers over the pH range of 6 to 13. The facilitated diffusion phenomenon in the dissolution of carboxylic polymers differs from that in membrane and biological systems in that the transport of polymer chains is not directly facilitated by any carriers. Proton-carriers facilitate the diffusion of hydrogen ions away from the polymer interface. As the concentration of hydrogen ions at the polymer interface decreases, the polymer solubility at the interface increases significantly, leading to a substantial increase in the polymer concentration driving force and, hence, the diffusion rate. A homogeneous chemico-diffusion model that elucidates the effects of the solution $\mathrm{pH}$, the concentration and acidity of carriers, and the polymer acidity on the facilitated diffusion was developed. Good agreement between experimental and theoretical results was achieved. There are optimum values of the carrier's $p K_{a}$, and of the solution $\mathrm{pH}$ which give a maximum facilitation effect. As the diffusion rate of the polymer is increased by the carrier, the overall polymer dissolution process changes from diffusion-limited to disentanglement-limited. () 2005 American Institute of Chemical Engineers AIChE J, 51: 415-425, 2005

Key words: facilitated diffusion, carboxylic polymer dissolution, coupled chemico-diffusion model, transitional Reynolds number, optimal $p K_{a}$ and $p H$ values
\end{abstract}

\section{Introduction}

Carboxylic polymers have been widely used in the pharmaceutical industry for drug controlled-release (Langer and Wise, 1984). The controlled-release drug delivery industry is estimated to have revenues of \$20 billion a year with excellent prospects for continued growth (Breslow et al., 2003). A novel application of the controlled-release using carboxylic polymers is the fused chemical reaction system for the remediation of wax precipitation, and deposition problems in subsea pipelines (Nguyen et al., 2001, 2004; Singh and Fogler, 1998). Wax precipitation and deposition occurring during production and transportation of crudes, espe-

Correspondence concerning this article should be addressed to H. S. Fogler at sfogler@umich.edu.

Current address of D. A. Nguyen: Research and Development Centre for Petroleum Processing, Petrovietnam, Vietnam; e-mail;anhduc@rdcpp.com.vn.

(C) 2005 American Institute of Chemical Engineers cially in deeper water and further away from the shore, is responsible for yearly losses of millions of dollars (Moritis, 2001). The primary challenge in clearing the pipeline blockages is to supply heat to regions further down the pipeline (for example, 70 kilometers) that are more susceptible to wax deposition (Brown et al. 1995). In the fused chemical reaction system, a polymeric coating surrounds the acid catalyst in a mixture of aqueous $\mathrm{NH}_{4} \mathrm{Cl}$ and $\mathrm{NaNO}_{2}$ reactants. As the polymer dissolves in the solution, the encapsulated acid catalyst is released and initiates the exothermic reaction. This exothermic reaction releases the heat necessary to melt and dissolve the paraffin deposit (Nguyen et al., 2003). Because the dissolution of the polymer determines the time and amount of the drug release (Ozturk et al., 1988a), or the location of the heat release (Nguyen et al., 2001), a thorough understanding of the kinetics, mechanism, and rate-limiting steps of the dissolution of carboxylic polymers in aqueous solutions is essential. 
The dissolution kinetics of carboxylic polymers have been studied by using either Dacron bags (Heller et al., 1978; Heller and Trescony, 1979) or polymer-coated glass plates (Spitael and Kinget, 1977; Spitael, Kinget et al., 1980) with uncontrolled stirring. The dissolution was speculated to be limited by either polymer diffusion or proton-transfer throughout the entire regime without any proof. Furthermore, no comprehensible mechanism for the dissolution of carboxylic polymers in aqueous solutions has been proposed.

Mooney et al. (1981a,b) successfully modeled the dissolution of solid monoacids in aqueous solutions in the absence and presence of buffer solutions using a chemico-diffusion model. Ozturk et al. (1988a) applied the Mooney's chemico-diffusion model for the dissolution of polyvinyl acetate phthalate (PVAP). However, no direct comparison between experimental and calculated polymer dissolution rates was shown. There are two main concerns in their method of calculation for the polymer dissolution rate. First, the rate-limiting step of the dissolution was not established in order to validate the use of the chemico-diffusion model. Moreover, it was assumed that the solubility and diffusivity of the polymer follow the same trend with those of monoacids, which is not at all the case! Therefore, Ozturk's approach of directly applying the Mooney's model to the polymer dissolution is not correct.

The main objective of this article is to provide a comprehensive mechanistic model for the dissolution of carboxylic polymers in aqueous solutions, emphasizing on the facilitated diffusion effects of the carriers. Studying the facilitated diffusion effects can also help understand the mechanism behind the accelerated dissolution of photoresists in the presence of carboxylic acids (Reiser, 1989).

The analysis of the effects of the facilitation carriers on the polymer dissolution is guided by the classic article of Schultz et al. (1974). This article reviewed conceptual models for the carrier-mediated transportation in membrane systems and provided a unified, theoretical framework for defining or characterizing certain invariant global aspects of carrier-mediated transport systems. However, polymer-solution systems are different from the membrane systems they studied in several important areas. First, carboxylic polymers ionize, producing hydrogen ions at the polymer interface. Therefore, both the polymer chains and hydrogen ions must diffuse from the polymer interface to the bulk solution. Second, because polymer chains are macromolecules with very low diffusivities, it is not advantageous to directly facilitate the diffusion of the polymer chains. Instead, because carboxylic polymers are more soluble at low hydrogen ion concentrations, we can introduce protoncarriers to facilitate the diffusion of hydrogen ions away from the polymer interface. The facilitated diffusion of hydrogen ions would increase the polymer solubility at the polymer interface substantially, thereby increasing the polymer diffusion rate significantly. Third, because hydrogen ions and hydroxyl ions are always in equilibrium, there is always a reverse flux of hydroxyl ions from the bulk solution to the polymer interface, countering with that of the hydrogen ions. More importantly, unlike in membrane systems, the hydrogen ion concentrations at the polymer interface and in the bulk solution are not independent in polymer-solution systems. Finally, as the polymer diffusion rate is increased significantly by the addition of proton-carriers, a change in the rate-limiting step may occur in the polymer dissolution process.

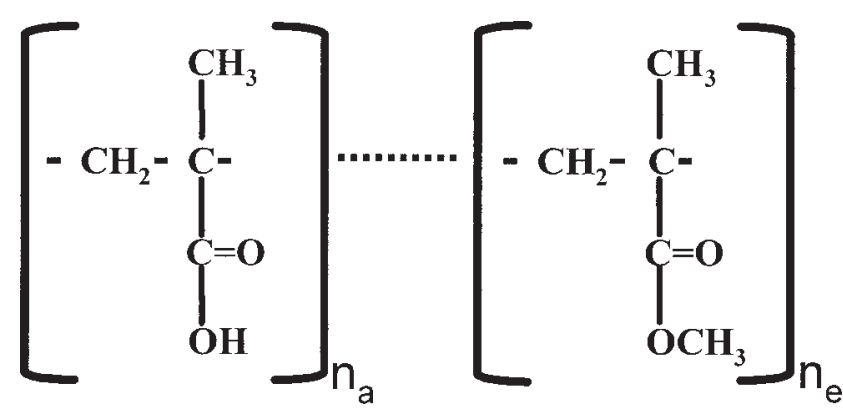

Figure 1. Eudragit type $\mathrm{L}$ and $\mathrm{S}$ Polymer structure.

The ester/acid group ratio $\left(n_{e} / n_{a}\right)$ is approximately 2 for Eudragit type S, and 1 for Eudragit type L. $n_{a}$ and $n_{e}$ (groups/ molecule) are the number of acidic and ester groups in a polymer molecule.

The change in the rate-limiting step in polymer dissolution processes is of great importance and interest in controlledrelease systems, because better control of the substance being released would be obtained if the polymer dissolution is not mass-transfer-limited. Therefore, in this work, we will investigate the effects of solution $\mathrm{pH}$, type and concentration of proton-carriers, and type of polymers on the kinetics and the rate-limiting step of the polymer dissolution. We also propose and verify experimentally a mechanistic model for the dissolution of carboxylic polymers in aqueous solutions.

\section{Materials and Methods \\ Materials}

Ammonium chloride $\left(\mathrm{NH}_{4} \mathrm{Cl}\right)$, sodium acetate $\left(\mathrm{CH}_{3} \mathrm{COONa}\right)$, sodium carbonate $\left(\mathrm{NaHCO}_{3}\right)$, sodium phosphate monobasic $\left(\mathrm{NaH}_{2} \mathrm{PO}_{4}\right)$, and lithium chloride $(\mathrm{LiCl})$ purchased from $\mathrm{Al}$ drich were used as carrier salts. Diluted hydrochloric acid $(\mathrm{HCl})$ was used to reduce the solution $\mathrm{pH}$, while sodium hydroxide $(\mathrm{NaOH})$ pellets were used to increase the solution $\mathrm{pH}$ of the solution. Both chemicals were supplied by Fisher Scientific. Eudragit type S-100 and type L-100 polymers in powder form supplied by RohmAmerica ${ }^{\circledR}$ were the carboxylic polymers analyzed in this project. Their structure is shown in Figure 1. Eudragit type L and S polymers are copolymers of methacrylic acid and methacrylic esters in different ratios. The ratio of the free carboxylic groups to the ester groups is approximately 1:1 in Eudragit type L, and about 1:2 in Eudragit type $\mathrm{S}$. Therefore, Eudragit type L polymer is more acidic than Eudragit type S polymer. The molecular weight of one monomer group containing a carboxylic group $\left(\mathrm{Mw}_{\mathrm{m}}\right)$ is about 286 Da for Eudragit type S, and 186 Da for Eudragit type L. Porsch et al. (2000) used size-exclusion chromatography to obtain the polydispersity index $\left(\mathrm{M}_{\mathrm{w}} / \mathrm{M}_{\mathrm{n}}\right)$ of the two polymers of approximately 2 . The average molecular weight is $135,000 \mathrm{Da}$ (Porsch et al., 2000; Rhom, 1996). Both polymers have an apparent $\mathrm{pK}_{\mathrm{a}}$ value of approximately 6.0 .

\section{Solubility measurements}

The solubility of the polymers was determined as follows: 20 $\mathrm{cm}^{3}$ of aqueous solutions at various $\mathrm{pHs}$ and excess amounts of the polymer film were put into series of vials. The vials were moved back and forth with a lateral displacement of $1 \mathrm{~cm}$ at a frequency of 100 cycles $/ \mathrm{min}$. Samples were taken at periods of 
time. The $\mathrm{pH}$ of the solution was measured using a $\mathrm{pH}$ meter (Cole-Parmer Scientific), while the polymer concentration was determined by using a Variant ${ }^{\circledR}$ UV spectrophotometer (Shimadzu). Equilibrium was ascertained to be attained.

\section{Polymer dissolution rate measurement}

The apparatus (Figure 2), was based on the rotating disk method described in Levich (1962), and consisted of an antifreeze-jacketed reactor maintained at $25 \pm 0.1^{\circ} \mathrm{C}$, with a ColeParmer constant-temperature water bath and a rotating disk. The dissolution medium $(500 \mathrm{~mL})$ was adjusted to an ionic strength of $0.5 \mathrm{~mol} / \mathrm{L}$ with $\mathrm{NaCl}$. The medium was placed into the reactor into which were immersed: a Cole-Parmer $\mathrm{pH}$ electrode and a glass disk holder with a shaft.

The polymer solution was prepared by dissolving $10 \mathrm{~g}$ polymer powder in a mixture of $97 \mathrm{~cm}^{3}$ ethanol and $3 \mathrm{~cm}^{3}$ water until a clear solution is obtained. The solution was then cast on 2-inch glass disks and let evaporate at slowly elevated temperatures to avoid bubble forming. The polymer-coated disks were finally heated at $70^{\circ} \mathrm{C}$ for $2 \mathrm{~h}$ to ensure all solvents have evaporated before being glued to the glass disk holder. The shaft and disk holder were rotated by a Pine Research Co. ${ }^{\circledR}$ (for rotation speed of 100 RPM), or a Yamoto ${ }^{\circledR}$ (for rotation speed greater than 100 RPM) overhead synchronous motor of variable speed; both were calibrated using a tachometer. The $\mathrm{pH}$-electrode was connected to a Cole-Parmer $\mathrm{pH}$ meter, which continuously monitored the $\mathrm{pH}$ of the medium during a dissolution run. The titrant (same solution but at higher $\mathrm{pH}$ ) was manually delivered into the medium to maintain a predetermined $\mathrm{pH}$ within that medium. Over the course of an experiment, the volume change from the added titrant was less than $1 \%$ of the initial volume. The Variant ${ }^{\circledR}$ UV spectrophotometer was set to measure the polymer concentration in the bulk solution at the optimum wavelength of absorbance for each polymer and solution (around $206 \mathrm{~nm}$ for Eudragit polymer type L, and around $210 \mathrm{~nm}$ for Eudragit polymer type S). In each case, the solution used for the dissolution run served as its own reference in the spectrophotometer.

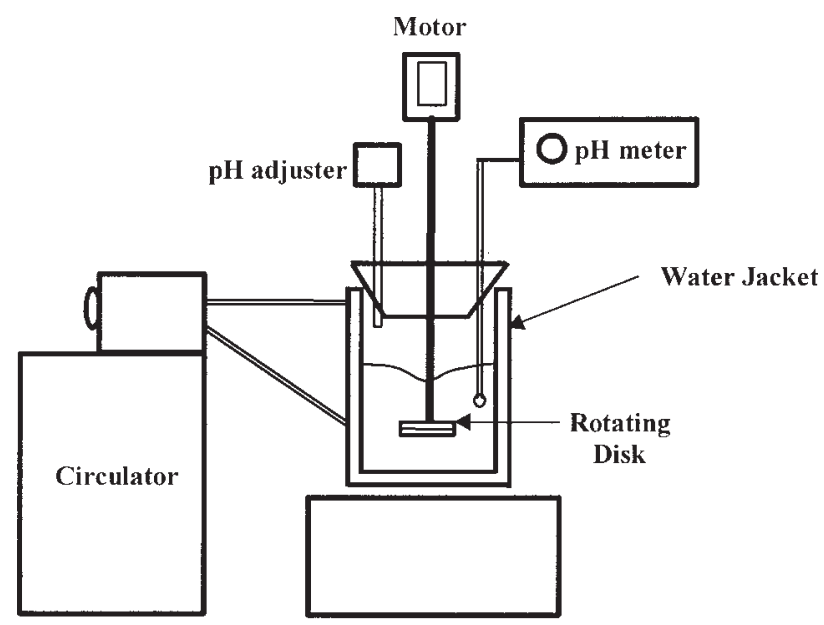

Figure 2. Rotating disk apparatus for polymer dissolution studies.

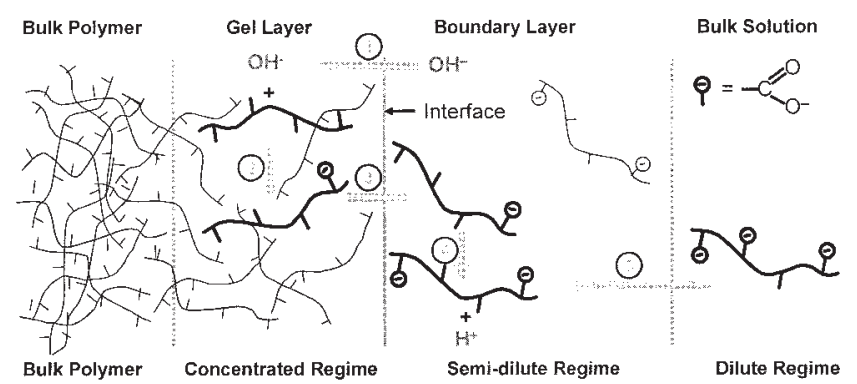

Figure 3. Dissolution mechanism of carboxylic polymers, circled numbers denote corresponding steps in the mechanism.

\section{Theoretical Analysis}

\section{Polymer dissolution mechanism}

Our XRD results show that both polymers type $\mathrm{L}$ and $\mathrm{S}$ are in an amorphous state while our DSC experiments on the polymer films reveal that the glass-transition temperature of the polymers is in the range of $70-90^{\circ} \mathrm{C}$. Therefore, the polymer films exist in a glassy state at room-temperature. The molecular weight of the polymers used in this work is approximately 135,000 Da (Rhom, 1996), an order of magnitude larger than the entanglement molecular weight (the molecular weight between entanglements - about 10,000 Da (Haward and Young, 1997)). Hence, in the polymer matrix, the polymer chains are entangled.

The dissolution mechanism of nonionic entangled glassy polymers has been studied extensively (Narasimhan and Peppas, 1996; Peppas, Wu et al., 1994). Nevertheless, for carboxylic polymers like the ones used in our work, the mechanism of polymer dissolution in aqueous solutions is different because it involves an ionization step that solubilizes the polymer chains. Therefore, in this work, the polymer dissolution mechanism is proposed to consist of the following steps (Figure 3):

(1) Diffusion of water and hydroxyl ions into the polymer matrix to form a gel layer.

(2) Ionization of polymer chains in the gel layer.

(3) Disentanglement of polymer chains out of the gel layer to the polymer-solution interface.

(4) Further ionization of polymer chains at the polymer interface.

(5) Diffusion of disentangled polymer chains away from the interface toward the bulk solution.

In the absence of a reaction kinetics rate limitation, step 1 is much faster than step 3 , because the diffusion of hydroxyl ions and water molecules is much faster than the disentanglement of polymer chains. Meanwhile, steps 2 and 4 (ionization of the polymer chains) are generally fast as observed during titration experiments. Thus, the polymer dissolution process can be either disentanglement-limited (step 3 ) if the polymer diffusion rate in the boundary layer is faster than the disentanglement rate, or diffusion-limited (step 5) if the diffusion rate is slower than the disentanglement rate.

\section{The disentanglement rate of polymer chains}

Narasimhan and Peppas (1996) derived the expression for the disentanglement rate of nonionic polymer chains as 


$$
\text { Rate }_{\text {Disentanglement }}=\frac{B}{\left(1-v_{1}\right)^{1.9}}
$$

where B is a constant that depends on the polymer molecular weight, solvent viscosity, and temperature, and $v_{1}$ is the solvent volume fraction.

In the disentanglement of ionic polymer chains, the solvent volume fraction $v_{1}$ depends on the hydrogen ion concentration in the gel layer. As the hydrogen ion concentration in the gel layer decreases, the degree of ionization of the polymer chains in the gel layer increases. Therefore, the polymer chains become more repulsive, creating a greater void fraction, resulting in a higher volume fraction of the solvent (that is, aqueous solution) in the gel layer. This increase in the solvent volume fraction $v_{1}$ leads to the increase in the disentanglement rate.

\section{The diffusion rate of polymer chains}

For simplicity, while still complying with the intent and purposes of this study, the following assumptions are made:

(1) All mass transfer resistances are lumped into a hypothetical stagnant liquid film (boundary layer) adjacent to the polymer interface.

(2) Reaction equilibrium are instantaneously established between all reactive species across the hypothetical stagnant liquid film.

(3) There is no current in the boundary layer.

The use of the idealized standard stagnant film model for analyzing mass transfer processes has been shown to predict the dissolution rates of solid acids and bases from rotating disks very well (Aunins et al., 1985; Mooney et al., 1981a,b). Therefore, the assumption of the hypothetical stagnant liquid film (assumption 1) is reasonable. Assumption 2 is sound, because the timescale for disentanglement and diffusion is much larger than that of ionization and association reactions. Moreover, because of the ionic strength of the solution, all diffusing ions are swamped with electrolyte. Therefore, no potential is built up due to differing diffusivities of various ions, and there are no electrodiffusive effects within the diffusion layer. Consequently, the zero-current constraint (assumption 3) is valid.

With those assumptions, the polymer diffusion rate (mol/ $\mathrm{cm}^{2} \cdot \mathrm{min}$ ) in rotating disk systems operated under laminar flow (Re less than $10^{4}$ to $10^{5}$ (Levich, 1962)), and sink conditions with the adjustment for the finite disk surface can be calculated as (Lehmkuhl and Hudson, 1971)

$$
J_{\text {Polymer }}^{\text {total }}=\frac{60 \mathrm{~s}}{1 \mathrm{~min}} \frac{1 \mathrm{dm}^{3}}{1000 \mathrm{~cm}^{3}} \frac{0.6205 D_{\text {Polymer }}^{2 / 3} \nu^{-1 / 6} \omega^{1 / 2}}{1+0.298\left(\frac{\nu}{D_{\text {Polymer }}}\right)^{-1 / 3}} C_{P s}
$$

\section{Accelerating the polymer diffusion rate}

From Eq. 2, an increase in the polymer diffusion rate in the presence of diffusion-promoters may be a result of a decrease of the solution viscosity, an increase of the diffusivity of polymer chains, or perhaps an increase of the polymer solubility at the polymer-solution interface. During the initial period in which the polymer dissolution rate is measured, the concentration of the polymer in the solution is minuscule. Consequently, viscosities of the solutions in the absence and presence

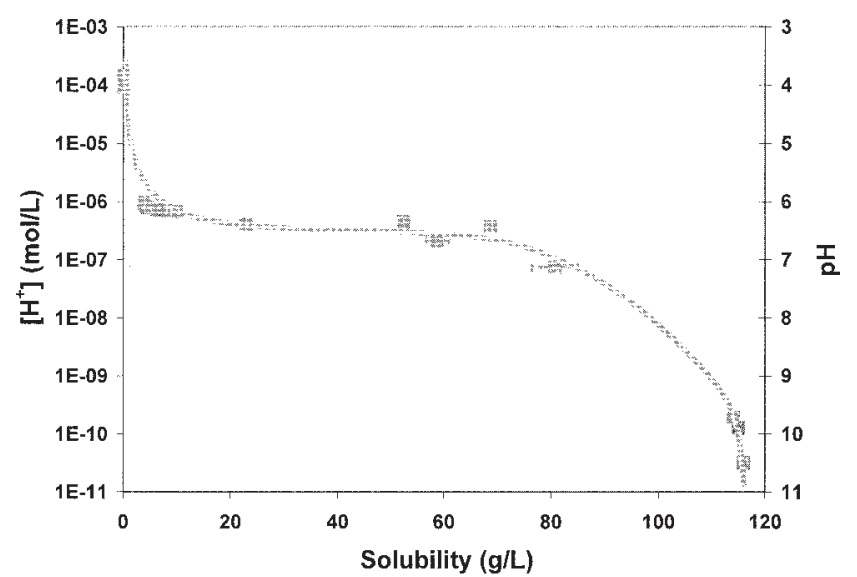

Figure 4. Solubility of the polymer as a function of the hydrogen ion concentration.

of diffusion-promoters are virtually identical. Because the $\mathrm{pH}$ and ionic strength of the bulk solution are constant, there is virtually no difference in the degree of ionization of the polymer chain, and consequently no difference in the Nernst potentials. Therefore, the diffusivities of the polymer chains are essentially unchanged in the presence of the diffusion-promoters (Daniel and Alexandrowicz, 1963; Katchalsky, 1954; Suzuki et al., 1969).

The only remaining term is the solubility of the polymer chains at the polymer interface. The solubility of the polymer chains increases significantly as the hydrogen ion concentration is decreased (Figure 4). Consequently, the major driving force for the substantial increases in the dissolution rate in the presence of the diffusion-promoters is the decrease of the hydrogen ion concentration at the polymer interface. During the dissolution process, hydrogen ions are produced at the polymer interface from the ionization of carboxyl groups on polymer chains (step 4 in Figure 3). The accumulation of hydrogen ions at the interface limits the extent of polymer chain ionization (cf. Eq. 7), thereby, inhibiting further solubilization of the polymer chains. Removal of these hydrogen ions at the interface increases the polymer dissolution rate. Therefore, in the dissolution of carboxylic polymers, proton-carriers can act as diffusion-promoters by enhancing the removal rate of hydrogen ions from the polymer interface toward the bulk solution, thereby increasing the diffusion rate of polymer chains from the interface.

Figure 5 depicts the reactions and diffusions in the boundary layer in the absence of any carriers. In event 1 , hydroxyl ions diffuse from the bulk solution to the polymer interface. Hydrogen ions are then removed from the polymer interface by two mechanisms: neutralization reaction with hydroxyl ions (event 2) and molecular diffusion down the hydrogen ion concentration gradient toward the bulk solution (event 3).

However, in the presence of a proton-carrier (Figure 6), hydrogen ions can also be removed from the polymer interface by reacting and diffusing with the proton-carrier (facilitated diffusion). Because the concentration of the carrier can be several orders of magnitude larger than that of hydrogen ions or hydroxyl ions, the carrier can greatly facilitate the diffusion of hydrogen ions away from the polymer interface. 


\section{Facilitated diffusion mechanism of hydrogen ions}

The facilitated diffusion of hydrogen ions follows a homogeneous chemico-diffusion model. In this model, hydrogen ions produced from the ionization reaction of disentangled polymer chains at the polymer interface reversibly react throughout the boundary layer with the dissociated form of the proton-carrier $\left(\mathrm{C}^{-}\right)$to form the undissociated form of the carrier (HC) as

$$
\begin{gathered}
H^{+}+C^{-} \rightleftarrows H C \\
{[H C]=\frac{\left[H^{+}\right]\left[C^{-}\right]}{K_{a}}}
\end{gathered}
$$

where $K_{a}(\mathrm{~mol} / \mathrm{L})$ is the acid dissociation constant of the carrier. The higher the value of $K_{a}$, the lower the affinity of the carrier with hydrogen ions.

The calculated concentration profiles of the three species $\mathrm{H}^{+}, \mathrm{C}^{-}, \mathrm{HC}$ in the presence of $0.05 \mathrm{~mol} / \mathrm{L}$ of the ammonia carrier are shown in Figure 6 with the arrows indicating the direction of net movement of these species. Hydroxyl ions diffuse from the bulk solution to the polymer interface in event 1. Hydrogen ions are removed from the polymer interface by three mechanisms. In the first mechanism, event 2 , hydrogen ions are consumed in the neutralization reaction with hydroxyl ions. In the second mechanism, event 3, hydrogen ions are removed by the molecular diffusion toward the bulk solution. In the third mechanism, event 4 , hydrogen ions react with the dissociated form of the carrier to form the undissociated form of the carrier (cf. Eq. 3). The undissociated form of the carrier then diffuses toward the bulk solution (event 5) where it

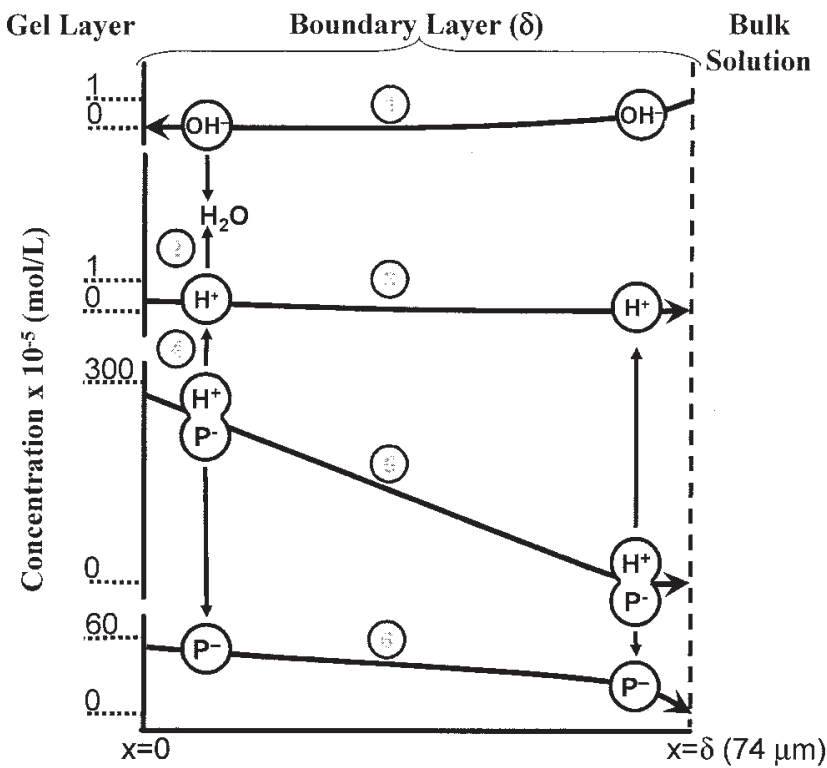

Figure 5. Reaction and diffusion in the boundary layer in the absence of any carriers.

Arrows show the direction of the concentration gradients, and of the diffusion processes.

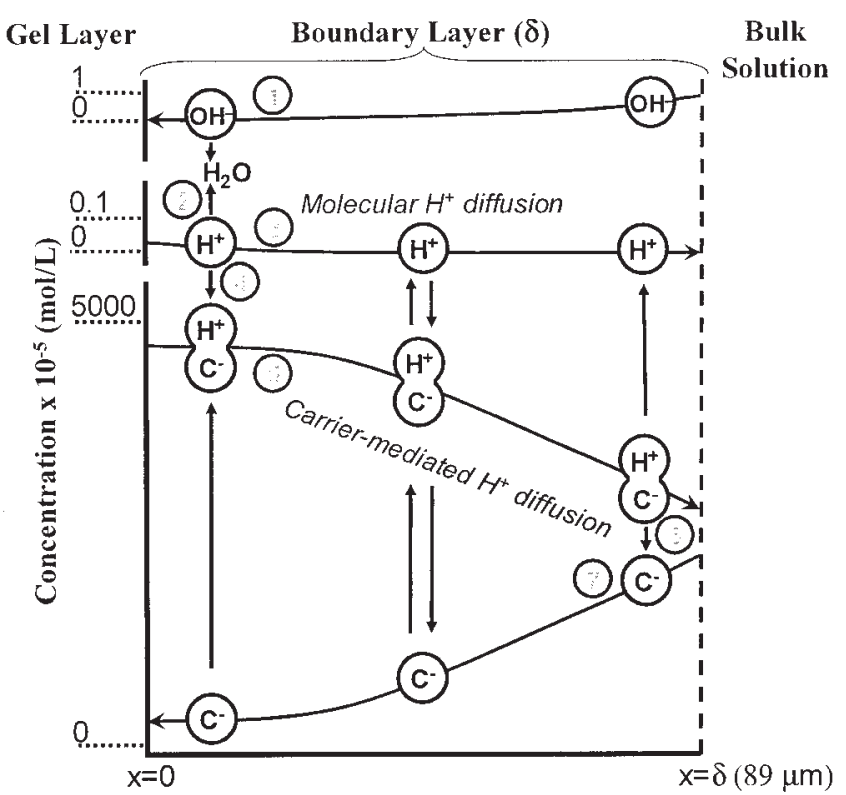

Figure 6. Mechanism of the faciliated diffusion of hydrogen ion in the boundary layer.

releases the hydrogen ion and the dissociated form of the carrier (event 6). In event 7, the dissociated form of the carrier diffuses from the bulk solution to the polymer interface. Hence, the proton-carrier acts as a shuttle, diffusing from the bulk solution to the polymer interface, binding with the hydrogen ion, carrying it across the boundary layer, and releasing it to the bulk solution. Proton-carriers can be any Brønsted-Lowry base (proton acceptor), such as $\mathrm{CH}_{3} \mathrm{COO}^{-}, \mathrm{NH}_{3}, \mathrm{NO}_{2}^{-}$, or $\mathrm{HPO}_{4}^{2-}$. Preferably, the carrier should have a structure that allows it to diffuse rapidly in aqueous solutions. The effectiveness of a carrier depends on its $\mathrm{pK}_{\mathrm{a}}$, and the solution $\mathrm{pH}$ as will be shown later.

\section{Effective facilitated diffusion factor}

Using the same concept as in the facilitated transport in membranes (Schultz et al., 1974), the hydrogen ion facilitation factor $\varphi_{H^{+}}$can be defined as

$$
\phi_{H^{+}}=\frac{J_{H^{+}, w \text { carrier }}^{\text {total }}-J_{H^{+}, \text {wlo carrier }}^{\text {total }}}{J_{H^{+}, \text {wlo carrier }}^{\text {total }}}
$$

where $J_{H^{+}}^{\text {total }}$ w/o carrier and $J_{H^{+}}^{\text {total }}$, carrier $\left(\mathrm{mol} / \mathrm{cm}^{2} \cdot \mathrm{min}\right)$ are the diffusion rate of hydrogen ions in the absence and presence of the carrier, respectively.

However, because the hydrogen ion concentration at the polymer interface can not be measured, the hydrogen ion facilitation factor can not be calculated directly. Because the facilitated diffusion of hydrogen ions results in the increase in the polymer diffusion rate, an effective facilitation factor $(\phi)$ can be defined using the polymer diffusion rates, which are directly measurable as

$$
\varphi \approx \frac{J_{\text {Polymer,w carrier }}^{\text {total }}-J_{\text {Polymer,w/o carrier }}^{\text {total }}}{J_{\text {Polymer,w/o carrier }}^{\text {total }}}
$$


where $J_{\text {Potal }}^{\text {tota }}$

and $J_{P o t a l}^{\text {total }}$

$\left(\mathrm{mol} / \mathrm{cm}^{2} \cdot \mathrm{min}\right)$

are the diffusion rates of polymer chains in the absence and presence of the carrier, respectively.

\section{Calculation of the polymer diffusion rate and the effective facilitation factor}

When the polymer dissolution is diffusion-limited, the polymer dissolution rate and the concentration profiles of all species in the absence and presence of the carrier were then calculated, based on the homogeneous chemico-diffusion model in a rotating-disk apparatus proposed by Mooney et al. (1981a,b). However, because the diffusivity of each species varies greatly, especially between the polymer chain and hydrogen ions, the boundary layer thickness was calculated as the sum product of the contribution of the flux and the Levich's boundary layer thickness of each species as in Aunins et al. (1985). To keep the model simple and still capture the most significant physical characteristics of the facilitated diffusion phenomenon, we neglected the effects of polydispersity and used the average molecular weight $M_{w}$ provided by Rohm America, and confirmed by Porsch et al. (2000) for both polymers in all calculations. Sample calculations of the polymer dissolution rate and the concentration profiles of all species across the diffusion layer in the absence and presence of a carrier are presented in Appendix B in Nguyen (2004).

The diffusivities of the hydrogen ion and hydroxide ion were fixed at $2.8 \times 10^{-5} \mathrm{~cm}^{2} / \mathrm{s}$ as in Mooney et al. (1981a). The carrier's diffusivity was assigned an average value of $5.0 \times$ $10^{-6} \mathrm{~cm}^{2} / \mathrm{s}$. Two different scenarios of the diffusivity of polymer chains were investigated and compared with experimental results. In the first scenario, the diffusivity of polymer chains was obtained by minimizing the deviation between calculated and experimental effective facilitation factors. The optimal polymer diffusivity found was $1.1 \times 10^{-6} \mathrm{~cm}^{2} / \mathrm{s}$. In the second scenario, the average value of the polymer diffusivity across the boundary layer was varied from $1 \times 10^{-9} \mathrm{~cm}^{2} / \mathrm{s}$ at a very low degree of ionization and polymer concentration $(\mathrm{pH} 6$, no carrier) to $1.3 \times 10^{-6} \mathrm{~cm}^{2} / \mathrm{s}$ at very high degree of ionization and polymer concentration ( $\mathrm{pH} 9,0.5 \mathrm{~mol} / \mathrm{L}$ ammonia carrier). While using a fixed polymer diffusivity makes the model more robust, the variable polymer diffusivity scenario is supported

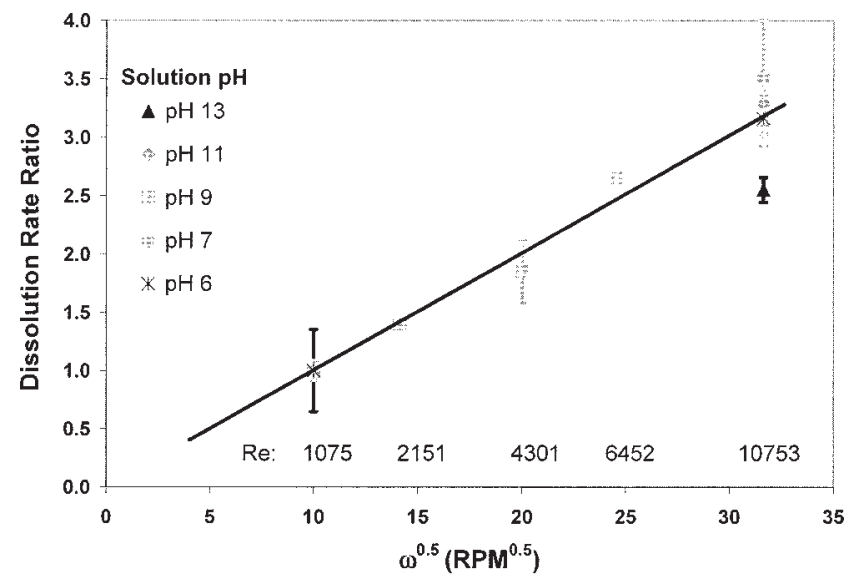

Figure 7. Effect of the rotating speed $\omega$ on the ratio of the dissolution rate at a given $\omega$ to the dissolution rate at $\omega=100 \mathrm{RPM}$ in the $\mathrm{pH}$ range 6-13.

by experimental results on the diffusion of ionic polymer chains.

The diffusivity of polymer chains was found to follow two different modes: a slow-diffusive mode where the polymer chains diffuse as clusters and a fast-diffusive mode where the polymer chains diffuse along with the counterions (hydrogen ions, sodium ions). Whether the slow-diffusive or fast-diffusive mode prevails depends on the concentration of the polymer, the degree of ionization of the polymer, and the ionic strength of the solution (Drifford and Dalbiez, 1985a,b; Katchalsky, 1954; Sedlak and Amis, 1992). The effective diffusivity of the slow-diffusive (cluster) mode is much lower than that of the fast-diffusive mode. For example, Drifford and Dalbiez (1985b) showed that the diffusivity of sodium polystyrene sulfonate (NaPSS) increases from $1 \times 10^{-9} \mathrm{~cm}^{2} / \mathrm{s}$ in the slow-diffusive mode to $2 \times 10^{-6}$ $\mathrm{cm}^{2} / \mathrm{s}$ in the fast-diffusive mode.

\section{Results and Discussion}

\section{Polymer solubility}

The solubilization process of carboxylic polymers can be represented as follows (Heller et al., 1978)
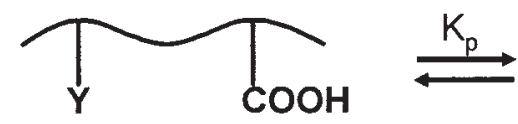

$$
K_{p}=\frac{\left[H^{+}\right]\left[P^{-}\right]}{[P H]}
$$

where $K_{p}(\mathrm{~mol} / \mathrm{L})$ denotes the average acid dissociation constant of a undissociated carboxylic group on the polymer chain $\left[\mathrm{P}^{-}\right](\mathrm{mol} / \mathrm{L})$, the concentration of dissociated carboxylate groups, and $[\mathrm{PH}](\mathrm{mol} / \mathrm{L})$ the concentration of undissociated carboxylic groups. 


\section{Polymer dissolution in the absence of any carriers}

When the diffusion of polymer chains is the rate-limiting step, the dissolution rate is directly proportional to the polymer solubility at the interface (cf. Eq. 2). Figure 7 shows the ratio of the dissolution rates (that is, ratio of the dissolution rate at a given $\omega$ to the dissolution rate at $\omega=100$ RPM) as a function of the square root of the rotation speed for different $\mathrm{pH}$ values between 6 and 13. The dissolution rate was found to increase linearly with the square root of the rotation speed over the $\mathrm{pH}$ range of 6-13, which is consistent with a diffusion-limited dissolution (cf. Eq. 2). Therefore, in the absence of any carriers and over the range of rotation speeds investigated, the dissolution process is limited primarily by the diffusion of polymer chains away from the polymer interface.

\section{Polymer dissolution in the presence of carriers: the existence of facilitated diffusion}

Figure 8 compares the polymer dissolution rate in the absence, and in the presence of the ammonia $\left(\mathrm{NH}_{3}\right)$ and acetate ion $\left(\mathrm{CH}_{3} \mathrm{COO}^{-}\right)$proton-carriers at $0.5 \mathrm{~mol} / \mathrm{L}$ and $\mathrm{pH}$ 9. At a rotation speed of $100 \mathrm{RPM}(\mathrm{Re}=1075)$, the polymer dissolution rate is still diffusion-limited in the presence of these carriers. As the rotating speed is increased further, the polymer dissolution rate changes to disentanglement-limited in the presence of the ammonia carrier. The polymer diffusion rate increases significantly (about 150 fold) in the presence of the ammonia carrier, but increases only 5 fold in the presence of the acetate ion carrier. The major difference between the two carriers lies in their $\mathrm{pK}_{\mathrm{a}}$ values. The ammonia carrier has a $\mathrm{pK}_{\mathrm{a}}$ value of 9.1, closer to the solution $\mathrm{pH}(9.0)$ than the $\mathrm{pK}_{\mathrm{a}}$ value of the acetate ion carrier (4.6). Consequently, the $\mathrm{pK}_{\mathrm{a}}$ value of

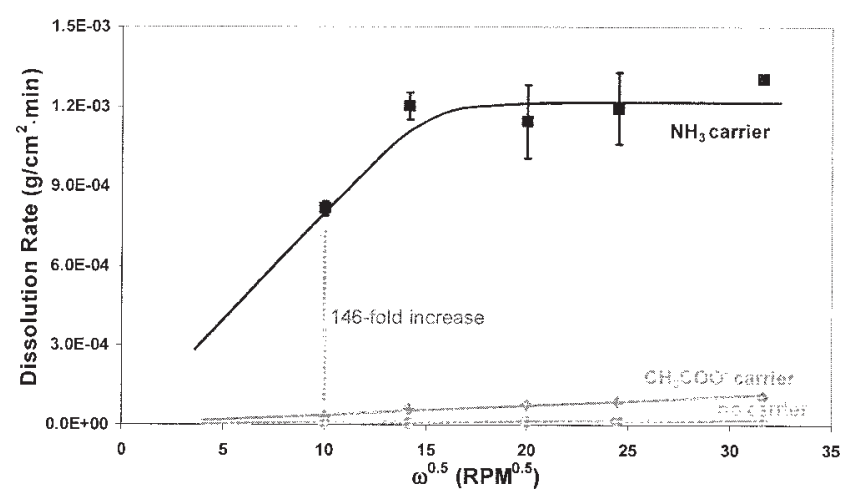

Figure 8. Facilitation effect of proton-carriers ([Carrier] $=0.5 \mathrm{~mol} / \mathrm{L}, \mathrm{pH}=9$ ).

the carrier plays a major role in the diffusion of polymer chains away from the polymer interface. The effect of the carrier acidity will be elucidated in a later section.

\section{Factors affecting the facilitated diffusion}

The analysis on the flux of polymer diffusion away from the polymer interface showed that the determining factor in the polymer diffusion rate is the concentration of hydrogen ions at the polymer interface. Thus, any factor which affects the interfacial hydrogen ion concentration should have a profound effect on the dissolution rate. Those factors include the concentrations of hydrogen ions and carriers in the bulk solution and the affinity of carriers and polymers with hydrogen ions.

Mole balances at the polymer interface in the absence and presence of the carrier (Appendix C in Nguyen (2004)) yields

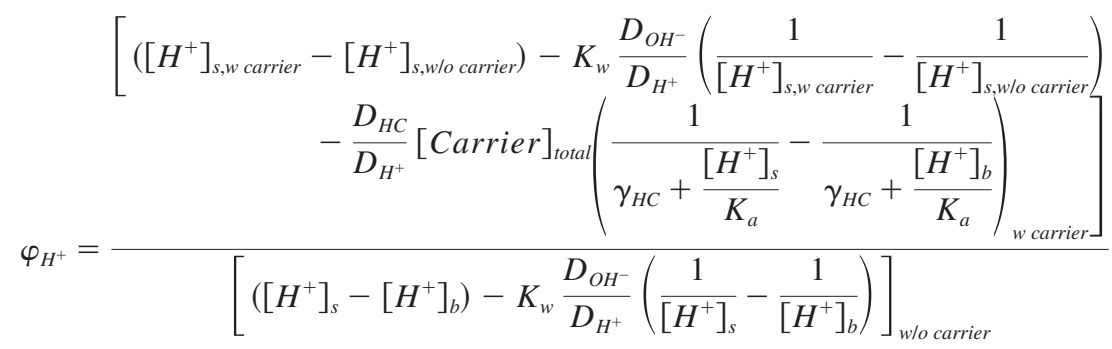

where, $\gamma_{H C}=D_{C^{-}} / D_{H C}, D_{H C}$ and $D_{C^{-}}\left(\mathrm{cm}^{2} / \mathrm{s}\right)$ are, respectively, the diffusivities of the undissociated and dissociated form of the carrier, $D_{H^{+}}\left(\mathrm{cm}^{2} / \mathrm{s}\right)$ the diffusivity of hydrogen ions, $D_{\mathrm{OH}^{-}}\left(\mathrm{cm}^{2} / \mathrm{s}\right)$ the diffusivity of hydroxyl ions $\left[\mathrm{H}^{+}\right]_{\mathrm{b}}$ and $\left[\mathrm{H}^{+}\right]_{\mathrm{s}}(\mathrm{mol} / \mathrm{L})$ the hydrogen ion concentrations in the bulk solution and at the polymer interface, respectively, and [Carrier $]_{\text {total }}(\mathrm{mol} / \mathrm{L})$ the dynamic total concentration of the carrier. A steady state mole balance on the carrier species shows that the dynamic total concentration of the carrier is constant throughout the system

$$
\begin{aligned}
{\text { [Carrier }]_{\text {total }}=[\mathrm{HC}]+} & \gamma_{H C}\left[\mathrm{C}^{-}\right] \\
& =[\mathrm{HC}]_{b}+\gamma_{H C}\left[\mathrm{C}^{-}\right]_{b}=\text { const }
\end{aligned}
$$

For a membrane where the hydrogen ion concentrations at the two boundaries are independent and there is no back flux of hydroxyl ions, Eq. 9 reduces to that found by Schultz et al. (1974) and Cussler (1997), that is

$$
\varphi_{H^{+}}=\frac{[\text { Carrier }]_{\text {total }} D_{H C}}{K_{a}\left(1+\frac{\left[H^{+}\right]_{b}}{K_{a}}\right)\left(1+\frac{\left[H^{+}\right]_{s}}{K_{a}}\right) D_{H^{+}}}
$$

Therefore, if the boundary layer in the polymer-solution system were similar to a membrane, one would expect the hydrogen ion facilitation factor to increase linearly with the carrier concentration, to go through a maximum as $\mathrm{K}_{\mathrm{a}}$ increases, and to 


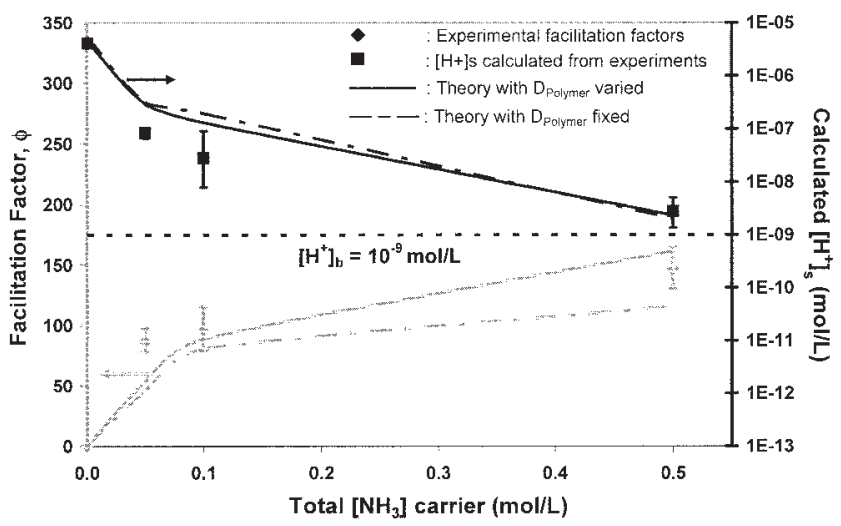

Figure 9. Effect of the concentration of ammonia carrier on the facilitation factor, and the concentration of hydrogen ion at the interface $(\mathrm{pH}=9)$.

decrease as $\left[\mathrm{H}^{+}\right]_{\mathrm{b}}$ increases. However, this is not the case for the polymer dissolution. In polymer-solution systems, the hydrogen ion concentration at the polymer interface depends on the hydrogen ion concentration in the bulk, the acidity and concentration of the carrier, and the acidity of the polymer, unlike in membrane systems. Therefore, Eq. 9 and Eq. 11 predict different trends as will now be shown.

\section{Effect of carrier concentration}

Figure 9 shows the effect of the concentration of the ammonia carrier $\left(\mathrm{NH}_{3}\right)$ on the diffusion rate of disentangled polymer chains away from the polymer interface at $\mathrm{pH}$ 9. Even at a small carrier concentration $(0.05 \mathrm{~mol} / \mathrm{L})$, the polymer diffusion rate was facilitated 87 fold, corresponding to a 49 fold decrease in the concentration of the hydrogen ions at the polymer interface. This significant decrease in hydrogen ion concentration reveals that the transport of hydrogen ions by coupling with the carrier dominates the molecular diffusion of hydrogen ions.

As the concentration of the carrier is increased in the bulk solution, more carriers are available to diffuse to the polymer interface, picking up more hydrogen ions at the interface. As a result, the hydrogen ion concentration at the polymer interface asymptotically approaches the hydrogen ion concentration in the bulk solution as the total concentration of the ammonia carrier in the bulk solution increases. Again, a lower hydrogen ion concentration at the polymer interface leads to a higher polymer solubility, a higher driving force, and a higher polymer diffusion rate (cf. Eq. 2). When the hydrogen ion concentration at the polymer interface approaches that in the bulk solution, fewer hydrogen ions are available to be removed. Therefore, the facilitation effect of adding more carrier decreased as shown in Figure 9 and as predicted by Eq. 9. Meanwhile, Schultz et al. (1974) and Cussler (1997) predicted a linear increase of the facilitation factor with the carrier concentration (cf. Eq. 11). Here again, the effect of the carrier concentration on the facilitation factor shows the difference in the facilitated diffusion between polymer-solution systems and membrane systems.

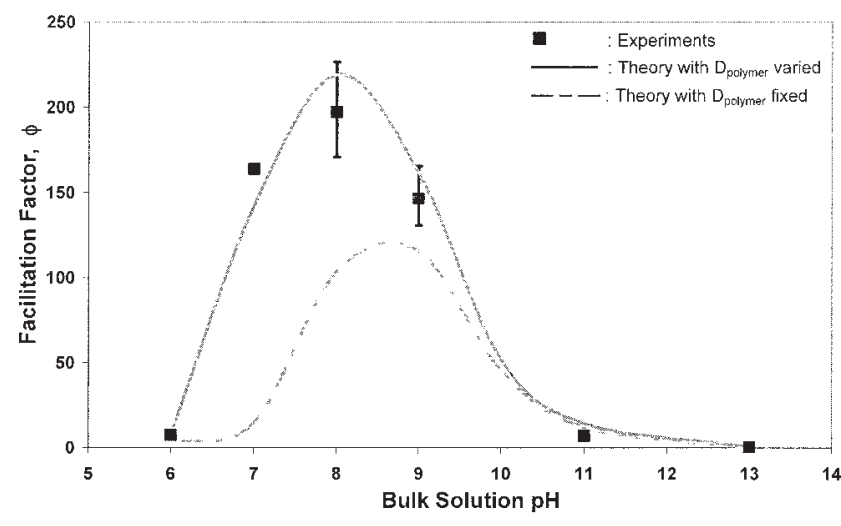

Figure 10. Effect of bulk solution $\mathrm{pH}$ on the facilitation factor $\left(\left[\mathrm{NH}_{3}\right]=0.5 \mathrm{~mol} / \mathrm{L}, \mathrm{pKa}=9.1\right)$.

\section{Effect of hydrogen ion concentration in the bulk solution}

As the bulk hydrogen ion concentration decreases, the interfacial hydrogen ion concentration also decreases. Therefore, the polymer solubility at the interface increases, leading to a higher polymer diffusion rate (higher effective facilitation factor). As previously noted, the determining factor in the polymer diffusion rate is the interfacial hydrogen ion concentration rather than the bulk hydrogen ion concentration. Moreover, facilitation by the ammonia carrier increases exponentially with the bulk solution $\mathrm{pH}$, reaching a maximum near the $\mathrm{pK}_{\mathrm{a}}$ of the carrier (9.1) then falls quickly at higher $\mathrm{pH}$ (Figure 10). The bell-shaped effect of the facilitation factor vs. $\mathrm{pH}$ will be explained in the later section.

\section{Effect of carrier acidity}

An exponential relationship was observed between the dissolution rate, and the $\mathrm{pK}_{\mathrm{a}}$ of the carrier up to the bulk solution pH of pH 9 (Figure 11), similar to what Spitael et al. (1977, 1980) observed. However, Figure 11 also shows that as the carrier's $\mathrm{pK}_{\mathrm{a}}$ is increased beyond 9, the facilitation factor reaches a maximum then falls rapidly to zero. Neither the Brönsted law approach suggested by Spitael et al. nor the buffer capacity in the microenvironment approach of Shek (1978) can explain the trends in Figure 11, which are explained

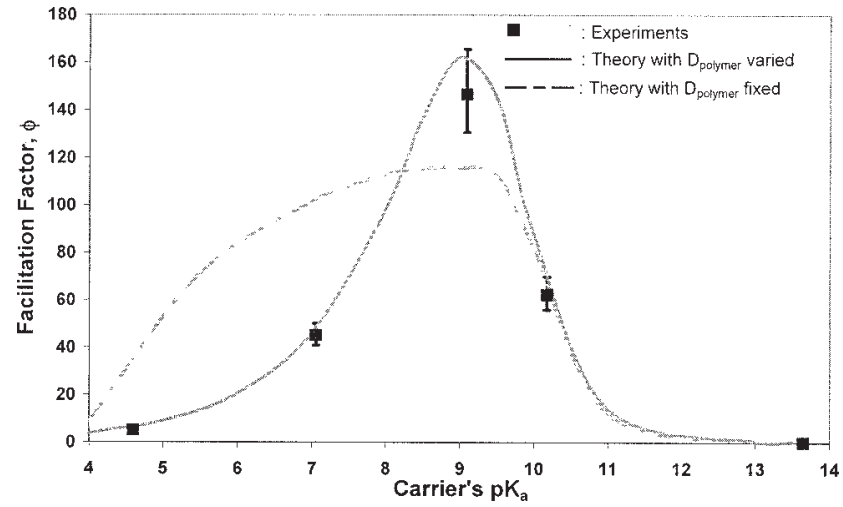

Figure 11. Effect of carrier's acidity on the facilitation factor ([carrier] $=0.5 \mathrm{~mol} / \mathrm{L}, \mathrm{pH}=9$ ). 
here. Moreover, both approaches consider the proton transfer between the carboxylic polymers and the carrier as rate-determining which contradicts with the fact that dissolution is diffusion-limited at a rotation speed of 100 RPM (Figure 7).

The effects of the solution $\mathrm{pH}$ and carrier's $\mathrm{pK}_{\mathrm{a}}$ on the facilitation factor can be explained by the mechanism in which proton-carriers facilitate the diffusion of hydrogen ions (Figure 6). In this mechanism, the carrier has two functions, namely, binding with a hydrogen ion at the polymer interface and releasing it in the bulk solution

$$
H^{+}+C^{-} \underset{\text { Releasing }}{\stackrel{\text { Binding }}{\rightleftarrows}} H C
$$

Both the binding and releasing abilities of a carrier depend on the solution $\mathrm{pH}$ and the $\mathrm{pK}_{\mathrm{a}}$ of the carrier (cf. Eq. 4).

Because the ionization reaction is fast, the concentrations of the hydrogen ions, the dissociated carrier $\left(\mathrm{C}^{-}\right)$, and the undissociated carrier (HC) are always in equilibrium (cf. Eq. 3). Therefore, the fraction of the undissociated carrier (HC) at the polymer interface $\left([\mathrm{HC}] /[\text { Carrier }]_{\text {total }}\right)_{s}$ reflects the number of moles of hydrogen ions that have been bound to one mole of the carrier at the polymer interface and is denoted as $f_{\text {releasing }}$. Meanwhile, the fraction of the dissociated carrier $\left(\mathrm{C}^{-}\right)$in the bulk solution $\left(\left[\mathrm{C}^{-}\right] /[\text {Carrier }]_{\text {total }}\right)_{b}$ represents the number of moles of hydrogen ions that have been released to the bulk solution by one mole of the carrier and is denoted as $f_{\text {releasing }}$. Hence, a higher $f_{\text {binding }}$ shows a greater ability of the carrier to bind with hydrogen ions. On the other hand, a higher $f_{\text {binding }}$ indicates the higher ability of the carrier to release hydrogen ions.

The hydrogen ion binding ability at the polymer interface and releasing ability in the bulk solution of the carrier can be obtained from Eqs. 4 and 10 when $\gamma_{H C}=1$ as

$$
\begin{aligned}
f_{\text {releasing }}=\left(\frac{\left[\mathrm{C}^{-}\right]}{[\text {Carrier }]_{\text {total }}}\right)_{b}=\frac{1}{1+\frac{\left[H^{+}\right]_{b}}{K_{a}}} & =\frac{1}{1+\frac{10^{-p H_{b}}}{10^{-p K_{a}}}} \\
= & \frac{1}{1+10^{p K_{a}-p H_{b}}}
\end{aligned}
$$

$$
\begin{array}{r}
f_{\text {binding }}=\left(\frac{[\mathrm{HC}]}{[\text { Carrier }]_{\text {total }}}\right)_{s}=\left(1-\frac{\left[\mathrm{C}^{-}\right]}{[\text {Carrier }]_{\text {total }}}\right)_{s} \\
=\frac{1}{1+10^{p H_{s}-p K_{a}}}
\end{array}
$$

Figure 12 shows that when $f_{\text {binding }}$ is high, $f_{\text {releasing }}$ is low and vice versa. In other words, when a carrier can bind with many hydrogen ions at the polymer interface, it can only release a few of them in the bulk solution and contrariwise. The combination of these two competing effects elucidates the bellshaped curves showing the dependence of the facilitation factor on the bulk solution $\mathrm{pH}$ (Figure 10) and on the carrier's $\mathrm{pK}_{\mathrm{a}}$ (Figure 11). At one extreme, where the $\left(\mathrm{pH}_{\mathrm{b}}-\mathrm{pK}_{\mathrm{a}}\right)$ and, therefore, $\left(\mathrm{pH}_{\mathrm{s}}-\mathrm{pK}_{\mathrm{a}}\right)$ are highly positive, $f_{\text {releasing }}$ is approximately 1 while $f_{\text {binding }}$ is 0 , the carrier can release hydrogen ions

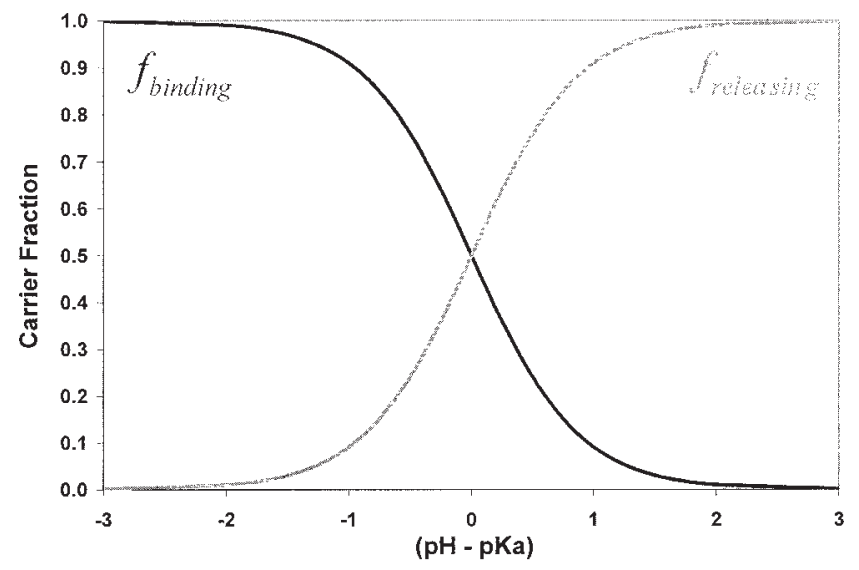

Figure 12. Effect of the difference between pH and carrier's pKa on the carrier functionality $\left(\gamma_{H C}=1\right)$.

in the bulk solution very well but can not bind with any hydrogen ions at the polymer interface. At the other extreme, where the $\left(\mathrm{pH}_{\mathrm{b}}-\mathrm{pK}_{\mathrm{a}}\right)$ and, therefore, $\left(\mathrm{pH}_{\mathrm{s}}-\mathrm{pK}_{\mathrm{a}}\right)$ are highly negative, the carrier can bind with hydrogen ions at the polymer interface very well but can not release any hydrogen ions in the bulk solution. Therefore, the facilitation effect is diminished or entirely eliminated in the extremes of very high or very low solution $\mathrm{pH}$ values (Figure 10 ) or $\mathrm{pK}_{\mathrm{a}}$ values (Figure 11). Figure 12 also shows that the facilitation factor will generally be the highest when $\left(\mathrm{pH}_{\mathrm{b}}-\mathrm{pK}_{\mathrm{a}}\right)$ is in the range of -1 to 1 , which is consistent with the experimental results in Figures 10 and 11. Because $\left(\mathrm{pH}_{\mathrm{b}}-\mathrm{pK}_{\mathrm{a}}\right)$ and $\left(\mathrm{pH}_{\mathrm{s}}-\mathrm{pK}_{\mathrm{a}}\right)$ are different, only follow the same trend, the maximum facilitation factor does not generally occur when the solution $\mathrm{pH}\left(\mathrm{pH}_{\mathrm{b}}\right)$ equals the carrier's $\mathrm{pK}_{\mathrm{a}}$.

For the facilitated diffusion in membrane systems, Schultz et al. (1974) also predicted that the facilitation factor increases as $\mathrm{K}_{\mathrm{a}}$ increases to up to $\left[\mathrm{H}^{+}\right]_{\mathrm{b}}$, then decreases as $\mathrm{K}_{\mathrm{a}}$ continues to increase (cf. Eq. 11). However, their result, Eq. 11, predicts that the facilitation factor decreases as $\left[\mathrm{H}^{+}\right]_{\mathrm{b}}$ increases instead of the bell-shaped effect shown in Figure 10. The reason is, again, for membrane systems, $\left[\mathrm{H}^{+}\right]_{\mathrm{b}}$ and $\left[\mathrm{H}^{+}\right]_{\mathrm{s}}$ are independent, whereas $\left[\mathrm{H}^{+}\right]_{\mathrm{s}}$ depends on $\left[\mathrm{H}^{+}\right]_{\mathrm{b}}$ and other system parameters in polymer-solution systems.

\section{Effect of polymer acidity}

In the absence of any carriers, the polymer dissolution is limited by the diffusion away of polymer chains from the polymer interface. Therefore, at the polymer interface, there is an accumulation of polymer chains leading to an accumulation of hydrogen ions. Because one mole of Eudragit type L polymer contains more carboxylic groups than one mole of Eudragit type $\mathrm{S}$ polymer, it releases more hydrogen ions at the polymer interface, leading to a lower polymer solubility. Therefore, the dissolution rate of Eudragit type L polymer is lower than that of Eudragit type $\mathrm{S}$ polymer in the absence of any carriers (Figure 13). However, as $0.5 \mathrm{~mol} / \mathrm{L}$ of the ammonia carrier is added to the solution, facilitating the diffusion of hydrogen ions away from the polymer interface, the hydrogen ion concentrations at the polymer interface are reduced to that of the bulk solution in both cases. Because Eudragit type L 


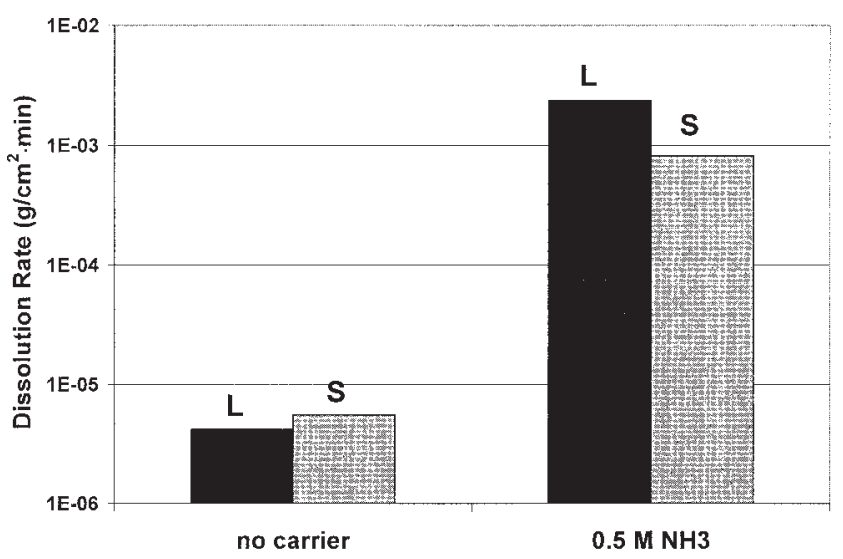

Figure 13. Effect of polymer acidity on the dissolution rate $(\mathrm{pH}=9)$.

polymer is more acidic than Eudragit type $\mathrm{S}$ polymer, it is more soluble at the same hydrogen ion concentration. Therefore, the dissolution rate of Eudragit type L polymer is three times larger than that of Eudragit type $\mathrm{S}$ polymer in the presence of 0.5 $\mathrm{mol} / \mathrm{L}$ of the ammonia carrier (Figure 13).

\section{Significance of facilitated diffusion}

One observes from Eq. 2 that as the rotation speed $\omega$ (or the Reynolds number) increases, the diffusion rate of the disentangled polymer chains from the interface to the bulk solution increases. When the diffusion rate is higher than the disentanglement rate, the polymer dissolution process changes from diffusion-limited to disentanglement-limited. Figure 7 shows that in the absence of a carrier the transition does not occur even at a very high rotation speed $(\omega=1,000$ RPM or $\operatorname{Re}=10753)$. However, as the total concentration of the ammonia carrier in the bulk solution increases, the polymer diffusion rate is significantly increased, leading to a much lower transitional Reynolds number where the changeover in the rate-limiting step occurs (Figure 14).

Therefore, the advantages of facilitated diffusion are twofold. First, it changes the polymer dissolution rate from diffusion-limited to disentanglement-limited, making the dissolution rate independent of flow conditions, thereby increasing the ability to control the polymer dissolution rate. Second, it

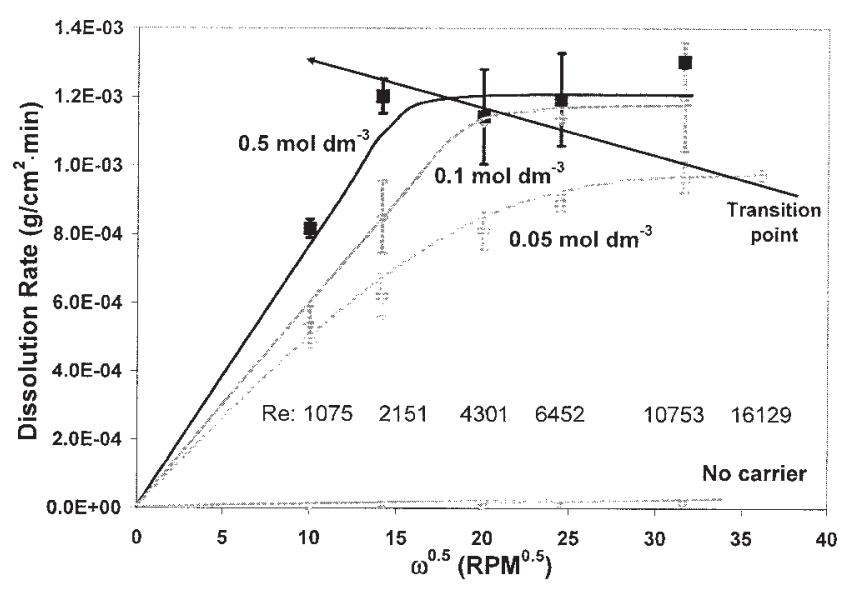

Figure 14. Change of the rate-limiting step $\left(\mathrm{NH}_{3}\right.$ carrier).

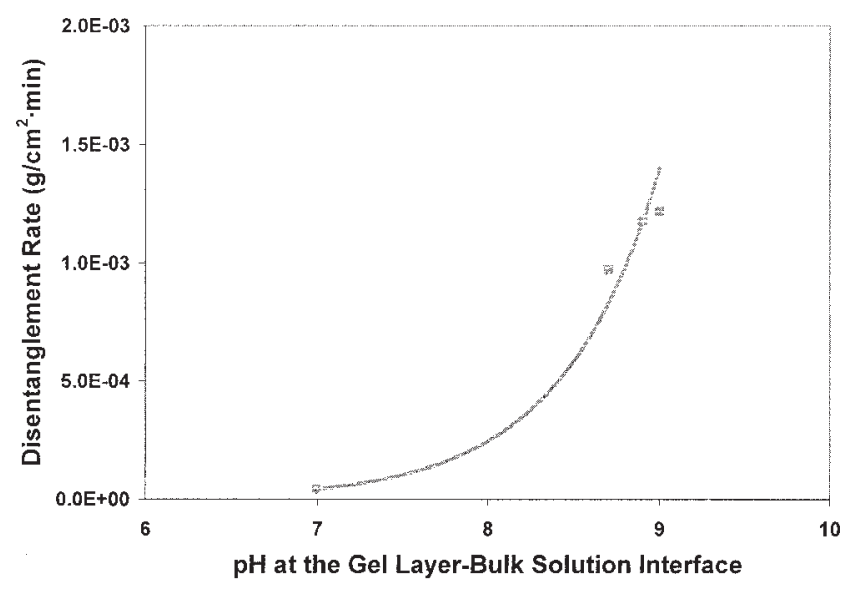

Figure 15. Effect of the interfacial pH on the disentanglement rate.

greatly increases the dissolution rate of low-solubility polymers or drugs in solution, thereby enhancing the dissolution of polymers and the efficiency of drugs.

\section{Disentanglement rate}

In the dissolution of carboxylic polymers, the disentanglement rate increases with an increase in the concentration of the carrier (Figure 14) or the bulk solution pH. Both effects can be explained by the decrease in the interfacial hydrogen ion concentration as the carrier's concentration or the bulk solution $\mathrm{pH}$ increases. As the hydrogen ion concentration in the gel layer decreases, the degree of ionization of the polymer chains in the gel layer increases. Therefore, the polymer chains become more repulsive, creating a greater void fraction, resulting in a higher volume fraction of the solvent (that is, aqueous solution) in the gel layer. This increase in the solvent volume fraction leads to the increase in the disentanglement rate (cf. Eq. 1). As a first approximation, the disentanglement rate can be correlated with the interfacial hydrogen ion concentration as (Figure 15)

$$
\begin{aligned}
\text { Rate }_{\text {Disentanglement }}=2.79 & \times 10^{-10} 10^{0.76 \mathrm{pHs}} \\
& =\frac{2.79 \times 10^{-10}}{\left[\mathrm{H}^{+}\right]_{s}^{0.76}}=9.6\left[\mathrm{OH}^{-}\right]_{s}^{0.76}
\end{aligned}
$$

\section{Conclusions}

The rate of polymer dissolution was increased significantly by the addition of proton-carriers over the $\mathrm{pH}$ range of 6 to 13 This significant variation in the polymer dissolution rate with $\mathrm{pH}$, concentration, and acidity of carriers is due primarily to the change in the concentration of hydrogen ions at the polymer interface. A homogeneous chemico-diffusion model was used to predict the polymer dissolution rate and concentration profiles of all species across the diffusion boundary layer. Good agreement between experimental and theoretical results was achieved. The facilitated diffusion of hydrogen ions by protoncarriers, which dominates the molecular diffusion of hydrogen ions, results in an increase in the rate of diffusion of polymer chains away from the polymer interface. The facilitated diffu- 
sion effect increases significantly with an initial increase in the carrier concentration, then approaches a limit at high carrier concentration. However, there are optimum values of the carrier's $\mathrm{pK}_{\mathrm{a}}$ and of the solution $\mathrm{pH}$, which give a maximum facilitation effect. The existence of the maxima is explained by the dual-functionality of the carrier, namely binding with the hydrogen ions at the polymer interface and releasing them in the bulk solution. The existence of different $\mathrm{pK}_{\mathrm{a}}$ optima for different $\mathrm{pH}$ values suggests the combination of several carriers to make the polymer dissolution rate independent of the bulk solution $\mathrm{pH}$ which is very much desirable in controlled-release applications. As the diffusion rate of the polymer chains away from the polymer interface is greatly facilitated by the carrier, the overall polymer dissolution process changes from diffusion-limited to disentanglement-limited. The transitional Reynolds number where the changeover in the rate-limiting step occurs decreases as the carrier concentration is increased, and is independent of the bulk solution $\mathrm{pH}$. Therefore, better control of the polymer dissolution rate in flow conditions could be obtained when enough carriers is present in the solution.

The existence of facilitated diffusion also presents a challenge to conventional practices to study the dissolution of either polymers or drugs in the pharmaceutical industry. For example, Heller et al. (1978; 1979) used Dacron bags and Spitael et al. $(1977 ; 1980)$ used polymer-coated glass plates to obtain the dissolution rate of polymers with uncontrolled stirring. This work illustrates that the external flow characteristics (Reynolds number), the nature and concentration of ions in the solution, and the bulk solution $\mathrm{pH}$ have very significant effects on not only the overall rate, but also the rate-limiting step of the polymer dissolution. In an extreme case, the polymer dissolution rates in the absence and presence of $0.5 \mathrm{~mol} / \mathrm{L}$ of ammonia at pH 8 are 200-fold different (cf. Figure 10).

\section{Literature Cited}

Aunins, J. G., M. Z. Southard, R. A. Myers, K. J. Himmelstein, and V. J. Stella, "Dissolution of Carboxylic-Acids.3. The effect of Polyionizable Buffers," J. Pharm. Sci., 74(12), 1305(1985).

Breslow, R., M. V. Tirrell, J. K. Barton, M. A. Barteau, C. R. Bertozzi, R. A. Brown, A. P. Gast, I. E. Grossmann, J. M. Meyer, R. W. Murray, P. J. Reider, W. R. Roush, M. L. Shuler, J. J. Siirola, G. M. Whitesides, P. G. Wolynes, and R. N. Zare, Beyond The Molecular Frontier : Challenges for Chemistry and Chemical Engineering, National Academies Press, Washington, DC (2003).

Brown, T. S., V. G. Niesen, and D. D. Erickson, "Measurement and Prediction of the Kinetics of Paraffin Deposition," J. Petrol Technol., 47(4), 328 (1995).

Cussler, E. L., Diffusion : Mass Transfer in Fluid Systems, 2nd. ed., Cambridge University Press, New York (1997).

Daniel, E., and Z. Alexandrowicz, "Sedimentation and Diffusion of Polyelectrolytes. 2. Experimental Studies with Poly-1-lysine Hydrohalides," Biopolymers, 1(5), 473 (1963).

Drifford, M., and J. P. Dalbiez, "Dynamics of Polyelectrolyte Solutions by Light Scattering," J. of Physique Lettres, 46L (1985a).

Drifford, M., and J. P. Dalbiez, "Effect of Salt on Sodium Polystyrene Sulfonate Measured by Light-Scattering," Biopolymers, 24(8), 1501(1985b).

Haward, R. N., and R. J. Young, The physics of Glassy Polymers, $2^{\text {nd }}$, ed., Chapman \& Hall, London (1997).

Heller, J., R.W. Baker, R. M. Gale, and J. O. Rodin, "Controlled Drug Release by Polymer Dissolution.1. Partial Esters of Maleic-Anhydride Copolymers-Properties and Theory," J. Appl Polym Sci., 22(7), 1991 (1978).
Heller, J., and P. V. Trescony, "Controlled Drug Release by Polymer Dissolution.2. Enzyme-Mediated Delivery Device," J. Pharm. Sci., 68(7), 919 (1979).

Katchalsky, A., "Problems in the Physical Chemistry of Polyelectrolytes," J. Polym Sci., 12(67), 159 (1954).

Langer, R. S., and D. L. Wise, Medical Applications of Controlled Release, CRC Press, Boca Raton, FL (1984).

Lehmkuhl G. D., and J. L. Hudson,"Flow and Mass Transfer Near an Enclosed Rotating Disk - Experiment," Chem. Eng. Sci. 26(10), 1601 (1971).

Levich, V.G., Physicochemical Hydrodynamics, Prentice-Hall, Englewood Cliffs, NJ (1962).

Mooney, K. G., M. A. Mintun, K. J. Himmelstein, and V. J. Stella, "Dissolution Kinetics of Carboxylic-Acids.1. Effect of ph Under Unbuffered Conditions," J Pharm Sci., 70(1), 13 (1981a).

Mooney, K. G., M. A. Mintun, K. J. Himmelstein, and V. J. Stella, "Dissolution Kinetics of Carboxylic-Acids.2. Effect of Buffers," J. Pharm Sci., 70(1), 22 (1981b).

Moritis, G., "Flow Assurance Challenges Production from Deeper Water," Oil \& Gas J. 1(1), 66 (2001).

Narasimhan, B., and N. A. Peppas, "On the Importance of Chain Reptation in Models of Dissolution of Glassy Polymers," Macromolecules, 29(9), 3283 (1996).

Nguyen, A. D., "The use of Fused Chemical Reactions to Remediate Paraffin Plugging in Subsea Pipelines," PhD Thesis, University of Michigan, Ann Arbor (2004).

Nguyen, A. D., H. S. Fogler, and F. F. de Moraes, "Fused Chemical Reactions. 3. Controlled Release of a Catalyst to Control the Temperature Profile in Tubular Reactors," Ind. Eng. Chem. Res., 43(18), 5862 (2004).

Nguyen, A. D., H. S. Fogler, and C. Sumaeth, "Fused Chemical Reactions. 2. Encapsulation: Application to Remediation of Paraffin Plugged Pipelines," Ind. Eng. Chem. Res., 40(23), 5058 (2001).

Nguyen, A. D., A. M. Iwaniw, and H. S. Fogler, "Kinetics and Mechanism of the Reaction between Ammonium Ion and Nitrite Ion: Experimental and Theoretical Studies," Chem. Eng. Sci., 58, 4351(2003).

Ozturk, S. S., B. O. Palsson, B. Donohoe, and J. B. Dressman, "Kinetics of Release from Enteric-Coated Tablets," Pharmaceut. Res., 5(9), 550 (1988a).

Peppas, N. A., J. C.Wu, and E. D. Vonmeerwall, "Mathematical-Modeling and Experimental Characterization of Polymer Dissolution," Macromolecules. 27(20), 5626 (1994).

Porsch, B, I. Hillang, A. Karlsson, and L. O. Sundelof, "Ion-Exclusion Controlled Size-Exclusion Chromatography of Methacrylic AcidMethyl Methacrylate Copolymers," J. of Chromatography A., 872(1-2), 91 (2000).

Reiser, A. Photoreactive Polymers, Wiley, New York (1989).

Rhom, Specifications and Test Methods for Eudragit ${ }^{\circledR}$ L 100 and Eudragit ${ }^{\circledR}$ S 100 (1996).

Schultz, J. S., J. D. Goddard, and S. R. Suchdeo, "Facilitated Transport via Carrier-Mediated Diffusion in Membranes.1. Mechanistic Aspects, Experimental Systems and Characteristic Regimes," AIChE J. 20(3), 417 (1974).

Sedlak, M., and E. J. Amis, "Concentration and Molecular-Weight Regime Diagram of Salt-Free Polyelectrolyte Solutions as Studied by LightScattering, "J. Chem. Phys., 96(1), 826 (1992).

Shek, E., "Buffer Capacity, not Buffer Catalysis, Affects the Dissolution Rate of Cellulose Acetate Phthalate," Pharmaceutical Industry., 40(9), 981 (1978).

Singh, P., and H. S. Fogler, "Fused Chemical Reactions: The Use of Dispersion to Delay Reaction Time in Tubular Reactors," Ind. Eng. Chem. Res., 37(6), 2203 (1998).

Spitael, J., and R. Kinget, "Factors Affecting the Dissolution Rate of Enteric Coatings," Pharmaceutical Industry, 39 (5), 502 (1977).

Spitael, J., R. Kinget, and K. Naessens, "Dissolution Rate of Cellulose Acetate Phthalate and the Bronsted Catalysis Law," Pharmaceutical Industry, 42(8), 846 (1980).

Suzuki Y, Noda I, and M. Nagasawa, "Diffusion of Polyelectrolyte in Presence of Added Salt," J Phys Chem-Us., 73(4),797 (1969).

Manuscript received Aug. 20, 2003, and revision received June 5, 2004. 